\title{
Understanding Accelerator Driven System (ADS) Based Green Nuclear Energy: A Review
}

\author{
Mohammad Kamal Hossain*, Md. Abu Taher, Mithun Kumar Das \\ Department of Physics, Comilla University, Comilla, Bangladesh \\ Email: ${ }^{*}$ kcu47@yahoo.com
}

Received 15 November 2014; accepted 25 October 2015; published 28 October 2015

Copyright (C) 2015 by authors and Scientific Research Publishing Inc.

This work is licensed under the Creative Commons Attribution International License (CC BY). http://creativecommons.org/licenses/by/4.0/

(c) $\underset{\mathrm{EY}}{\mathrm{i}}$ Open Access

\section{Abstract}

This paper reflects the scopes of accelerator driven system (ADS) based nuclear energy, as a reliable source of electric energy generation, comparing to the other existing non-renewable and renewable sources. There are different limitations in the use of every source of electric energy but in consideration of minimum environmental impact, exclusively inherently low greenhouse gas (GHG) emission, and also, high life time with maximum power production efficiency, nuclear would be the best choice. From this study it was found that several difficulties involved in the ADS based energy production, more specifically, difficulties regarding the target parameters, coding system, waste management, etc. Hence suggestions from this study points out that if it is possible to ensure more energy efficient production of enriched uranium, improved nuclear fuels and reactors that allow greater utilization, extended life times for nuclear power plants (NPPs) that reduce the need to build new facilities, improved coding system capable of minimizing the discrepancy between theoretical and experimental calculation of spallation products, improved data library with sufficiently available high energy nuclear data to perform a better coding analysis, and finally, considering the environmental safety if the disposal of the radioactive wastes could manage more effectively, nuclear energy would then play a significant role in minimizing future energy crisis worldwide as well as to save our loving green earth.

\section{Keywords}

Green Nuclear Energy, Spallation, Accelerator Driven System, Transmutation of Wastes, Sustainable Energy Development

\footnotetext{
*Corresponding author.
} 


\section{Introduction}

Energy is central to economic growth. Higher living standards and increased energy consumption are intrinsically related. Fighting poverty worldwide will require a means of raising the world's living standards to levels the developed nations take for granted. This will involve the introduction of a basic energy infrastructure and consumption patterns [1]. Worldwide demand for energy is rapidly increasing and it is expected that it could double by 2050. Therefore it is important to develop and deploy multiple energy sources in the context of a comprehensive energy plan i.e., a broad mix of energy technologies is essential to meet the growing demand. At the same time, the evidence is clear that $\mathrm{CO}_{2}$ emissions must be reduced globally. Abundant, affordable, and environmentally responsible energy must be developed, both domestically and internationally, to meet that demand [2].

There is a range of energy resources, often called non-renewable and renewable energy resources, available to generate electric energy. Non-renewable sources of energy can be divided into two types: fossil fuels and nuclear fuel. Coal, oil and natural gas are fossil fuels. Among the renewable sources solar, wind, etc. play the dominating roles. Whatever the contribution to the total world energy is, all of them have some notable environmental limitations, individually. For instance, air quality is the primary environmental concern for coal-fired power plants, which emit a significant amount of air pollutants. Coal mining also can significantly affect the environment by polluting water and destroying wildlife habitats. The health effects from coal mining include death from cave-ins, coal dust explosions, methane explosions and methane inhalation. Many coal miners also have suffered lung ailments and other disabilities. Natural gas plants emit an amount of particulates, smog-forming pollutants and carbon dioxide. It is also responsible for the ground water pollution from the hydraulic fracturing process [3]. In addition to ecological disturbances from fossil fuel extraction, there are certain cultural consequences for communities around the Pacific Rim. These communities, many indigenous, are threatened by the depletion of specific resources they depend upon for their livelihoods and culture [4].

Most types of renewable energy sources create little or no air pollution or greenhouse gases. But the fact is that both wind and large-scale solar facilities can require significant land area. For example, a solar thermal plant uses between 5 (five) and 10 (ten) acres of land per megawatt of electricity generated. Water also can be a concern for certain solar thermal electric plants that, like conventional power plants, need water to generate electricity. Installing both solar and wind can disturb the natural environment, so wildlife studies are often conducted before projects proceed. Bird and bat migration and flight patterns are important considerations for wind turbines since poorly located turbines can cause excessive deaths [3]. Therefore, most of the environmental problems the world facing today—including climate change, air pollution, oil spills, and acid rain—result from our dependence on fossil fuels [4] and a little bit ecological disturbance that arises from the use of renewable energy sources. Growing concerns over the consequences of climate change may severely limit future access to fossil fuels. A forced choice between energy and environment could precipitate a major economic crisis, an environmental crisis, or both [1].

Among the non-renewable sources nuclear power has been used to produce electricity for public distribution since 1954 [5]. This option of electric power generation should be retained, precisely because it is an important carbon free source of power that can potentially make a significant contribution to future electric power supply [6]. But now it appears that hesitations in further developments of nuclear power are primarily due to the environmental concerns arising from the nuclear waste disposal schemes involving long-term geological storage of commercial reactor waste [7]. The possible solution that overcomes the above problem and ensures the future growth of nuclear power as a reliable, safe and abundant source of electricity is the incineration of long-lived transuranic elements (TRU) and transmutation of fission products (FP) which can make its disposal in geological repository environmentally acceptable. With a view to do so, several projects and programs are going on worldwide, in finding the possibility of separating (or partitioning) the long-lived radioactive waste from the used fuel and transmuting it into shorter-lived radionuclides so that the management and eventual disposal of this waste are easier, less expensive and will be environmentally friendly [8]. The above is done in an assembly equipped with the spallation arrangement and an accelerator driven sub-critical nuclear reactor system (ADS). The aim of this study is to understand the ADS based green nuclear energy as a part of understanding sustainable energy development in order to meet future energy needs as well as to save our green earth.

\section{Spallation and ADS}

Starting from the observations of particle cascades in cosmic rays interactions in 1930 scientist's attempt in in- 
vestigating the spallation yields is growing day to day but not been established yet. A theoretical picture of an incident particle colliding successively with several nucleons inside target nucleus, losing a large fraction of its energy was proposed by Serber in 1947. Before, in 1937 Weisskopf considered possibility of emission of neutron from excited target nucleus. In the end of fifties, Metropolis and Dostrovsky (who used the ideas of Serber and Weisskopf) suggested description of spallation as two-step process involving energy deposition and subsequent evaporation. They formulated and performed first Monte Carlo calculations of the reactions. Such treatment of spallation reactions is used from that time up to now [9]. As spallation applications require more precise knowledge, they are being investigated with the increasing interest in the last two decades while in recent years the world has registered obvious progress in the accelerator technique. The successful functioning of several high energy research accelerators (Berkeley, KEK), the construction of the LHC, ... brought new technologies also to mid- and low-energy accelerators. Also, advances like superconductivity for magnets and RF cavities, ion sources have led to the practical realization of high-power beams [10].

Next to fission and fusion, spallation is an efficient process for releasing neutrons from nuclei [11]. By definition it is a process in which a light projectile such as proton, neutron, or light nucleus with the kinetic energy from several hundreds of $\mathrm{MeV}$ to several $\mathrm{GeV}$ i.e., a high-energy hadron or a nucleus interacts with a heavy nucleus and causes the emission of a large number of hadrons mostly neutrons or fragments [12]. Therefore spallation is by far the most attractive means of neutron production when it comes to energy deposition per neutron. The spallation mechanism initiates with intra-nuclear cascade (INC) reactions, followed by de-excitation of nuclei through evaporation, multi-fragmentation and fission [13]. The first, so-called fast stage of the spallation is highly non-equilibrated process. High energy proton causes an intra-nuclear cascade on a time scale $\sim 10^{-22} \mathrm{~s}$. The incident projectile goes through the target nucleus and deposits a significant amount of excitation energy and angular momentum, while ejecting only a few high energy nucleons and, with a minor yield, pions and light ions. The result of the first stage is excited residual nucleus in thermodynamical equilibrium (totally or partly equilibrated), with excitation energy a few $\mathrm{MeV}$ /nucleon. The second, so-called slow stage of the spallation, consists in de-excitation of the residuum by evaporation of particles. The isotropic emission, in the system of nucleus, of nucleons (mainly neutrons), light and heavy ions (d, t, He, Li, Be, B, .., $\gamma$ ) takes place on a time scale $\sim 10^{-18}-10^{-16} \mathrm{~s}[9]$. Broadly, the different stages of spallation reactions are:

Fast Direct Process:

- Intra-Nuclear Cascade (nucleon-nucleon collisions)

Pre-Compound Stage:

- $\quad$ Pre-Equilibrium

- Multi-Fragmentation

- $\quad$ Fermi Breakup

Compound Nuclei:

- $\quad$ Evaporation (mostly neutrons)

- $\quad$ High-Energy Fissions

Inter-Nuclear Cascade

Low-Energy Inelastic Reactions

- $\quad(n, x n)$

- $\quad$ (n, nf), etc. [14]

Both the INC and de-excitation processes lead to the production of neutrons and spallation products as illustrated in Figure 1 [13]. Spallation products are of two types: spallation evaporation and spallation-fission products. The spallation products can be measured using two methods: direct or inverse kinematics. In direct kinematics, a relativistic light projectile hits a heavy target. The spallation products, which stop in the target, can be detected using $\gamma$-spectroscopy and mass spectrometry. This method has the possibility to measure the yields of the meta-stable states of residual nuclei, it can use radioactive targets, and it consumes less beam time. On the other hand, it is impossible to measure the yields of very long-lived, stable and very short-lived nuclei, and the off-line yields measurements are more time consuming. In the case of inverse kinematics, a relativistic heavy nucleus hit a light target. The spallation products leave the target in forward direction and can be immediately in flight using the appropriate experimental set-up [12].

The neutrons produced in spallation reactions can be characterized by their energy and spatial distributions and multiplicity. The neutron multiplicity will determine the current and beam energy of the proton-driver 


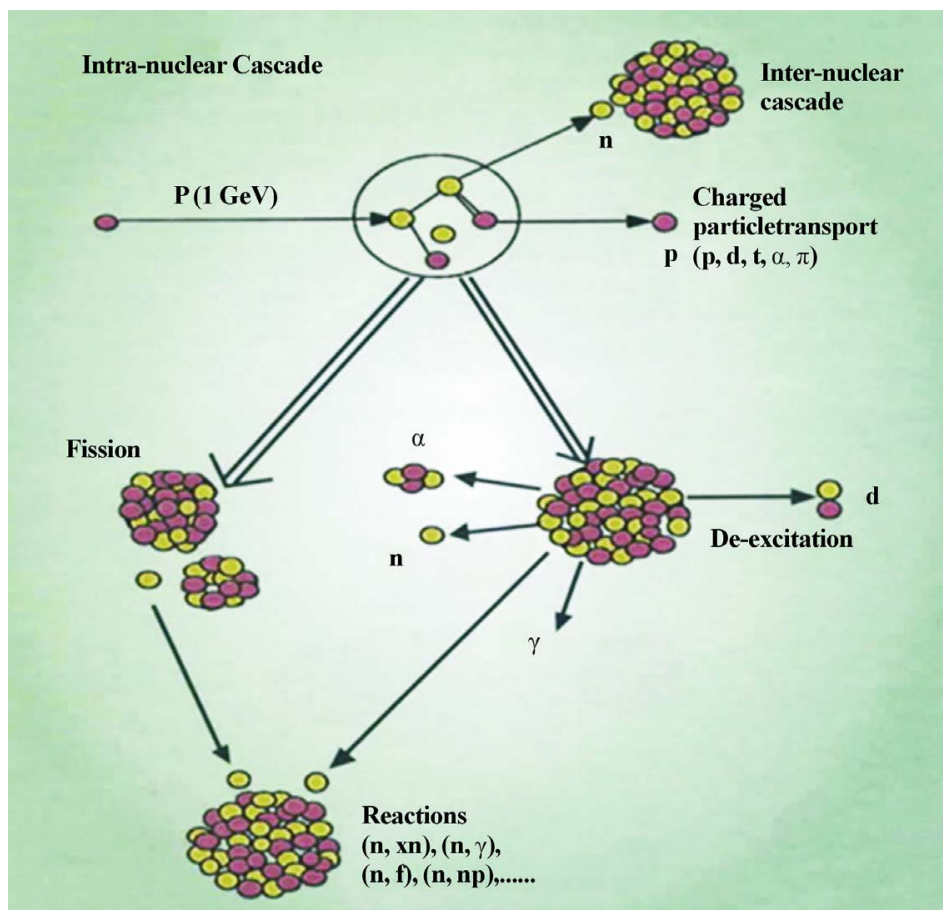

Figure 1. Spallation reaction and different stages with the products [14].

accelerator while their energy and spatial distribution shapes the geometry of the spallation target and the shielding to high-energy neutrons [15]. The spallation neutrons have only a very small probability of causing additional fission events in the target [8]. Spallation reactions do not only produce neutrons but also residual nuclei. Most of these nuclei are radioactive. In addition, the residual nuclei will contribute to the corrosion and to the radiation damages in the target, accelerator window and structural materials [15].

Neutrons from Spallation have huge potential in research in earth, in space, in medical industry, in energy production, etc. More clearly, they play a significant role in a wide domain of applications ranging from neutron sources for condensed matter, particle physics experiments, transmutation of nuclear waste, material studies, isotope production to astrophysics, simulation of detector set-ups in nuclear study and radiation protection around an accelerator and also in space, etc.

The long-term hazard of radioactive waste arising from nuclear energy production is a matter of continued discussion and public concern in many countries [10]. Transmutation of highly radioactive nuclear waste can be performed using an accelerator driven system (ADS), where high energy protons impinge on a spallation target to produce neutrons. These neutrons are multiplied in a sub-critical core, while simultaneously fissioning the minor actinides into short-lived or stable nuclides [13]. The accelerator driven system (ADS) has recently been receiving increased attention due to its potential to improve the flexibility and safety characteristics of transmutation systems [10].

The idea of the ADS was born in 90's with articles of C.D. Bowmam and C. Rubbia, who independently proposed a new approach to the problems of radioactive waste and limited uranium resources: to introduce extra neutrons produced in spallation reaction to the core of the subcritical reactor. A subcritical reactor is a nuclear fission reactor that produces fission without achieving criticality. Instead of sustaining a chain reaction, a subcritical reactor uses additional neutrons from an outside source. Such a reactor coupled to a particle accelerator to produce neutrons by spallation is called an ADS [10]. Therefore, it is said that, a sub-critical reactor is driven by an Accelerator-Driven System (ADS) to compensate for the loss of neutron economy which otherwise would have been self-sustaining in a critical reactor [13]. Figure 2 shows how the electric energy is generated using a subcritical reactor.

In particular, as the spallation target is surrounded by a blanket assembly of nuclear fuel, such as fissile isotopes of uranium or plutonium (or thorium-232 which can breed to U-233), there is a possibility of sustaining a fission reaction. This is described as an accelerator-driven system (ADS). In this system, the neutrons produced 


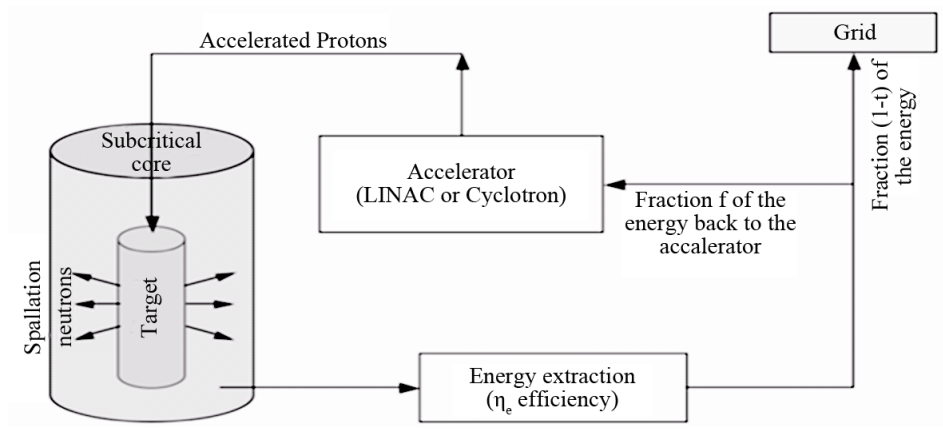

Figure 2. ADS based electric energy generation [14].

by spallation would cause fission in the fuel, assisted by further neutrons arising from that fission. Up to $10 \%$ of the neutrons could come from the spallation, though it would normally be less, with the rest of the neutrons arising from fission events in the blanket assembly. An ADS can only run when neutrons are supplied to it because it burns material which does not have a high enough fission-to-capture ratio for neutrons to maintain a fission chain reaction. One then has a nuclear reactor which could be turned off simply by stopping the proton beam, rather than needing to insert control rods to absorb neutrons and make the fuel assembly subcritical. Because they stop when the input current is switched off, accelerator-driven systems are seen as safer than normal fission reactors.

An ADS can be used to destroy heavy isotopes contained in the used fuel from a conventional nuclear reactor-particularly actinides. Here the blanket assembly is actinide fuel and/or used nuclear fuel. One approach is to start with fresh used fuel from conventional reactors in the outer blanket region and progressively move it inwards. It is then removed and reprocessed, with the uranium recycled and most fission products separated as waste. The actinides are then placed back in the system for further incineration. Figure 3 shows the different aspects of ADS products based on their (ADSs) physical characteristics.

ADS could also be used to destroy longer-lived fission products contained in spent nuclear fuel, such as ${ }^{99} \mathrm{Tc}$ and ${ }^{129} \mathrm{I}$ (213,000 and 16 million years half-lives, respectively). These isotopes can acquire a neutron to become ${ }^{100}$ Tc and ${ }^{130}$ I respectively, which are very short-lived, and beta decay to ${ }^{100} \mathrm{Ru}$ and ${ }^{130} \mathrm{Xe}$, which are stable [8].

It is therefore, can then be said that the term ADS comprehensively includes all non-self-sustaining fissioning neutron multiplying assemblies which are driven by an external neutron source provided by a charged particle accelerator and a neutron producing target. Worldwide, ADS systems under current study include both thermal and fast neutron multiplying media comprised of either liquid or solid (lattice) fuel and driven by either cyclotron or linear proton accelerators and spallation targets (liquid and solid) of various heavy metals. The underlying missions targeted for ADS systems span the range from nuclear waste incineration with ancillary power production through power production with integral waste self-incineration to finally, excess neutron production for the purpose of isotope production via neutron capture reactions on targets [16].

Another area of current interest in the use of ADS is in their potential to dispose of weapons-grade plutonium, as an alternative to burning it as mixed oxide fuel (MOX) in conventional reactors. Two alternative strategies are envisaged: the plutonium and minor actinides being managed separately, with the latter burned in ADS while plutonium is burned in fast reactors; and the plutonium and minor actinides being burned together in ADS, providing better proliferation resistance but posing some technical challenges. Both can achieve major reduction in waste radiotoxicity, and the first would add only 10\% - 20\% to electricity costs (compared with the oncethrough fuel cycle) [8].

\section{Parametric and Technological Considerations in Spallation and ADS Study}

The spallation target constitutes the physical and functional interface between the accelerator and the sub-critical reactor. For this reason it is probably the most innovative component of the ADS. The target design is a key issue to investigate in designing an ADS and its performances are characterized by the number of neutrons emitted for incident proton, the mean energy deposited in the target for neutron produced, the neutron spectrum and the spallation product distribution. The development and design of the target implies a detailed assessment of different aspects mutually interacting, from the physics of spallation reaction including neutron generation and 


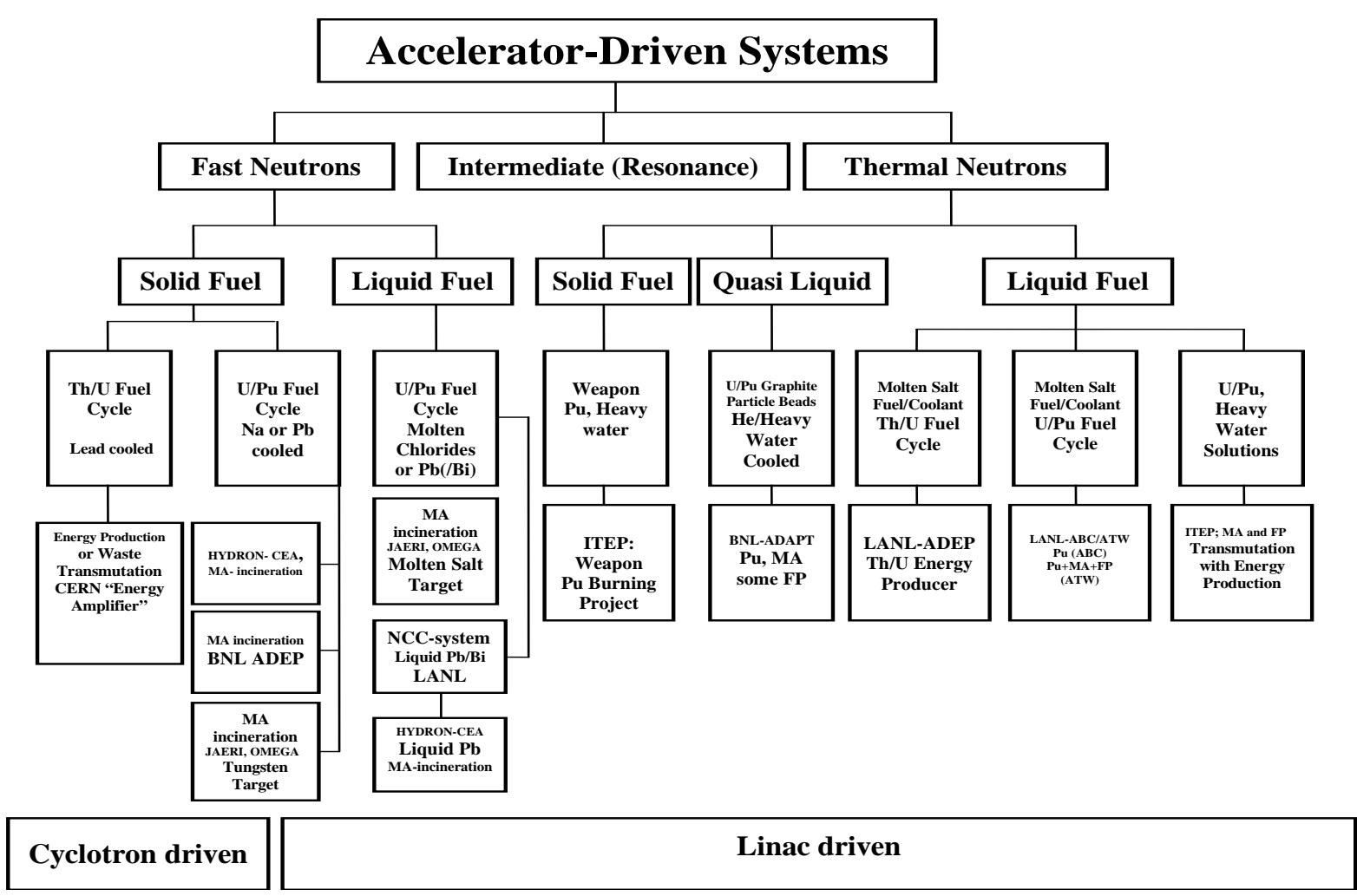

Figure 3. Classification of existing ADS concepts according to their physical features and final objectives [14].

distribution, spallation product yields and damage rates to technological issues, such as choice of the most suitable material, power density distribution, heat removal, thermo-mechanics and fabricability. In particular, accurate and rigorous assessment of nuclear parameters under different physical conditions is the prerequisite for an optimal design of the target and its interaction with the sub-critical core [17].

The physical study on the spallation target is one of the basic researches of the ADS design. From the point of view on the engineering application, the basic problems such as spallation neutron yield, energy spectrum and energy deposition will be firstly concerned. The neutron yield per incident proton is the most interesting data for the practical application in the ADS study, which is related with the incident proton energy and the target material [18]. Also, the neutron yield is an important consideration in selecting the energy and current parameters of the proton beam. Many experimental data show that for a given neutron yield, lower beam current is required at higher beam energy. An advantage of higher beam energy is greater penetration depth in the target, leading to lower axial power densities [19].

The ADS system utilizes the neutrons produced in a spallation target by a high-energy proton beam to drive a sub-critical reactor containing both fissionable fuel and radioactive waste [18]. The neutron fluxes produced in spallation reactions will depend strongly on the projectile-target combination. Specifically, it depends on the material properties, the kinetic energy of the projectile and the geometric configuration of the target [13]. In principle the heavier the target nucleus the larger the neutron excess and the larger the neutron yields [15]. The number of spallation neutrons per incident proton depends on the beam energy and on the mass of the target nuclei [19]. As because of the above, spallation target is ideally conceived to be high atomic number material and heavy density metals like lead, bismuth, mercury, uranium, tungsten, tantalum and lead-bismuth eutectic (LBE) to fit the requirement extremely well. The gain factor between heavy and light targets is around a factor of five. However, the radiotoxicity induced in the spallation target could be drastically reduced when using lighter targets [15].

The size of the target has a great influence in parameter consideration. Determination of the appropriate size of the target is so complicated. One must consider the design of the reactor, heat dissipation, the production process of the target, neutron yield and the amount of a combination of factors. The reaction mechanisms of dif- 
ferent neutron spectra and reactor materials are different. Consequently, the target of different sizes results in different energy spectra of neutrons, and thus matches with a different reactor. Therefore, a comparison of the neutron spectrum in the different target sizes is necessary [18]. Moreover, because of the requirement to minimize the size of the target in relation to the core dimensions to provide good neutron economy; the target design is driven to maximum power density. For the very high power densities reached in a spallation target (several hundred kW per liter for proton beam powers of several MW), a flowing liquid metal target material provides the best option for practical removal of the heat by convection. The use of a heavy liquid metal (HLM) also significantly reduces the damage caused by intense radiation to the target itself and the structural materials. However, the use of HLM gives rise to problems of erosion, corrosion, and liquid metal embrittlement (LME) of structural materials that are in contact with the circulating metal at its characteristic high temperatures and high fluid velocities. In addition, difficulties concerning the confinement of volatile spallation products and the separation between the proton beam vacuum and the liquid spallation target must also be addressed [19].

Both window and windowless target concepts are currently being investigated in ongoing ADS design projects worldwide. However, the constraints introduced by a window in terms of the required subcritical-core working temperature, the need of frequent window replacement, and/or the need for an extra cooling loop for the spallation target have raised major design challenges for this approach. These concerns are reinforcing an R\&D effort aimed at solving key design issues for the windowless target concept, namely compatibility of the beam line vacuum and target outgassing, and control of spallation products evolved from the target, and of HLM evaporated from the target surface. These R\&D efforts are not dedicated exclusively to supporting the windowless target concept, but also address the safety issues arising from a possible window rupture in the window target case [19].

Two severe operating conditions of the spallation target are the very high volumetric heat deposition rate and irradiation by high fluence of energetic protons and neutrons. Regarding the former, the peak power dissipation density in the target volume can be of the order of a few $\mathrm{kW} / \mathrm{cm}^{3}$. In such a case the use of heavy liquid metals (HLM) like lead and bismuth may serve both as spallation target and heat removing media. Also the problems associated with irradiation damage like swelling, cracking/embrittlement and other degradations can easily be avoided using such a target. However, these and corrosion/erosion issues need to be resolved with new and better materials for liquid target container and process equipment [7].

In order to get the full pledge benefit from the spallation dependent ADS technology it is important to consider the parametric factors having direct or indirect influence on the system. Among these, the parameters which characterize the spallation module are extremely important since they will have several impacts on the design of the whole ADS. Some important notes regarding this are:

- The neutron angular and energetic distribution will determine the transmutation potential of the system.

- Target with high neutron source strength will drive an ADS with lower multiplication factor, thus improving safety conditions.

- Costs saving can be achieved by using a target with high proton to neutron conversion factor. In this way fewer particles current is required.

Therefore, for achieving the best from an ADS assembly, the spallation module design should then be based on a balanced optimization between neutronic efficiency, material properties and thermo-hydraulic performances [17]. Moreover, in considerations of different issues related to the target, technology, efficiency and environmental safety, an ADS should also have the following attractive features:

- extremely high level of inherent safety;

- minimum production of long lived wastes;

- ability to transmute long-lived nuclear wastes generated in normal reactors and;

- more efficient use of abundantly available natural fuel $\left({ }^{232} \mathrm{Th}\right)$ [18].

All these four are directly dependent on the above three. Hence a combined attempt would provide the path to reach the goal. Butin the practical realization of the ADS, the most challenging task is the development of a high energy $(\sim 1 \mathrm{GeV})$ and high current $(\geq 10-15 \mathrm{~mA})$ proton accelerator, which is reliable, rugged and stable in order to provide uninterrupted beam power to the spallation target over long periods of time [7].

In spallation neutron sources and Accelerator Driven Systems, along with all, radiation protection has mainly to deal with specific problems induced by high-energy reactions in the target. This is the case, for instance, of the radioactivity in the target in which the spallation reactions create a much larger variety of radioactive isotopes than activation by low energy neutron fluxes. The existence of a high-energy tail in the spectrum of neu- 
trons generated in the spallation target is also of importance for the shielding of the facility [20].

Besides the above, it is better to add here the following that, research work on the spallation target design and ADS set up have been going on for a long periods of time since its concept given by Bowman and Rubbia. As a result, data available for different parameters obtained from different experimental observations may help in further parametric and technological research and development. Moreover, a large variety of existing nuclear data may provide the scope of reliable ADS design as it facilitates a detail study of the ADS assembly. For this reason some experimental findings are discussed here with a view to take it into considerations during future research and development so that it can then be made more efficient and reliable, also. For instance:

Both lead $(\mathrm{Pb})$ and lead-bismuth $(\mathrm{Pb}-\mathrm{Bi})$ exhibit very low neutron capture making them good candidates from a neutronic standpoint. Lead $(\mathrm{Pb})$ might at first appear a better target choice than LBE, because of its considerably lower production of ${ }^{210} \mathrm{Po}$ (a migratory $\alpha$-emitter) under neutron bombardment. However, lead has the critical disadvantage of a higher melting point $\left(327^{\circ} \mathrm{C}\right.$ compared with $\left.123.5^{\circ} \mathrm{C}\right)$, which presents severe thermo-mechanical design challenges for the target structural materials. The ${ }^{210} \mathrm{Po}$ release problem in LBE is mitigated by the fact that $\mathrm{Po}$ forms a chemical bond with $\mathrm{Pb}$ to form metastable Pb-Po, which has an emanation rate (vapour pressure) 1000 times lower than that of Po by itself. Therefore, Lead-bismuth eutectic (LBE) becomes today the reference target material for ADS applications [19].

Mercury $(\mathrm{Hg})$ has also been considered as a spallation target material because of its low melting point $\left(-38.9^{\circ} \mathrm{C}\right)$ and the absence of the ${ }^{210} \mathrm{Po}$ problem. However, its high volatility would seriously complicate the confinement of its radioactive isotopes, and its low boiling temperature would make its use in ADS difficult due to the high working temperature of the target and also of the subcritical multiplier. In addition its high neutron capture cross section degrades the leakage and therefore the neutron efficiency of the target [19].

The optimum proton energy for production of neutrons by spallation in a heavy metal target, in terms of costs, target heating, and system efficiency, lies in the range 600 to $1000 \mathrm{MeV}$. Two completely different kinds of machines can be considered for acceleration of high currents of protons to an energy of $600-1000 \mathrm{MeV}$ : linear accelerators (linacs) and cyclotrons. CW linear accelerators will be the baseline technology for XADS demonstration facilities and full-scale industrial ADS plants, because of their high potential for achieving the required level of beam reliability and availability, and also because of their power upgrade capability. Linac beam theory and recent technology advances have confirmed that a linac capable of delivering up to $100 \mathrm{MW}$ at $1 \mathrm{GeV}$ is a relatively direct extension of existing technology. For ADS applications involving a few tens of megawatts, HLM is the target-material of choice at present. This selection is principally driven by the need for high-neutron yield per proton, the high power deposition densities reached within the target, and compatibility of the target material with structural materials [19].

\section{Models and Codes in Spallation Study}

The detailed engineering design of an ADS will require that the performance of the spallation target and all the problems related to the existence of high-energy particles can be predicted with sufficient accuracy. Some of the important quantities to assess are:

- intensity and spatial distribution of the spallation neutron flux outside the target;

- radiation damage and gas production in target, window and structural materials;

- radiotoxicity, activity and corrosion problems inside the target;

- required shielding to neutrons with much higher energies than in conventional reactors.

Provision of ADS related data is mainly done by means of theoretical development and computational implementation of nuclear models. For ADS, cross sections for the important materials need to be known for all possible reaction channels. This total amount of required information is so large that experiments alone can never cover the nuclear data needs. To fill this gap, the nuclear data must be simulated computationally, with the help of theoretical nuclear reaction models. The models can then be used in areas where no measurements exist [21]. Also, designing and operating a spallation neutron source requires reliable high-energy transport codes. In these codes, the elementary cross-sections and characteristics of all produced particles are either obtained from nuclear data libraries or calculated by nuclear-physics models. For several decades spallation models have been developed aiming at reproducing the particle and the residue production [22]. Moreover, application of high energy accelerators for production of energy and radioactive nuclides as well as transmutation of nuclear waste is based on large scale using of spallation reactions for neutron generation in thick targets and following multip- 
lication of the neutrons in subcritical blankets [23]. As a result, neutron production calculations have to be as reliable as possible when designing a neutron spallation source. However, correct predictions of light charged particles (LCP), in particular tritium and helium, and residues in the spallation target and surrounding materials are important as well, most of the time for radioprotection and safety issues. As regards residues, $\beta / \gamma$ or $\alpha$-emitters, delayed neutron progenitors that can be produced in the target, in particular volatile elements in the case of liquid metals, are some examples of what must be properly estimated [22] and all of these are done by the help of different computer codes.

The development of a transmutation program based on the ADS requires accurate spallation simulation tools in order to design such systems. Correct description of such a complicated process as high energy particle interaction with matter can be performed by means of Monte Carlo method. It is the most suitable tool to take into account all details of nuclear reaction mechanism and particle transport both in high and low energy range [23]. But there are some limitations and the main limitations of the Monte Carlo method are:

- The correctness of the neutron cross-sections, but this is common to all transport codes.

- The physical model used, but for low energy neutron transport this is mainly expressed by the partial reaction cross-sections, double differential cross-sections, etc.

- Its intrinsic imprecision, due to the random nature of the events generated, though it may be reduced by increasing the number of trial events $\mathrm{N}$, now possible with the help of fast parallel computers which can generate many events simultaneously [10].

Several simulation codes and combinations of these codes exist. Now though there are some limitations in the existing codes there is a strong need of simulation codes for ADS assembly projection. Among the existing codes the two widely used transport codes used to simulate the spallation reactions are LCS (LAHET Code System) and FLUKA. The LCS code, developed at Los Alamos National Laboratory is a Monte Carlo code for treating the transport and interactions of nucleons, pions, muons, light ions and antinucleons in complex geometry. LAHET includes both the Bertini and the ISABEL intranuclear cascade model as user options. An evaporation model for the break-up of light nuclei is also included. An optional multistage pre-equilibrium model has been implemented as an intermediate stage between the intranuclear cascade and the evaporation phase of a nuclear reaction. Alternative level density parameterizations are also included. The FLUKA (FLUctuating KAscade simulation program) code, developed at CERN, is a Monte Carlo code able to simulate transport and interaction of electromagnetic and hadronic particles in any target material over a wide range of energies. FLUKA use the PEANUT (Pre-Equilibrium Approach to Nuclear Thermalization) model to describe an elastic nuclear interaction. This model consists of intranuclear cascade (INC), pre-equilibrium, evaporation and deexcitation. The current version of the code can simulate neutron interaction and transport down to thermal energies (multigroup below $20 \mathrm{MeV}$ ) and hadron-hadron and hadron-nucleus interactions up to $100 \mathrm{TeV}$ [17].

The neutronics behaviour of an ADS is an important subject to be studied in the development phase. Detailed ADS design studies call for an accurate 3D geometry model. The code MCNP in its standard shape can only be used for static calculations. However, the time-dependent behaviour of an ADS requires full 3D burnup analyses. In order to make these analyses possible the NRG OCTOPUS code system was extended to include the possibility of performing 3D burnup analyses with MCNP and a burnup code (ORIGEN or FISPACT). The version of MCNP which is used at NRG was substantially extended with important modifications for ADS applications. A useful addition for ADS analyses is the possibility to generate 3D reaction rates in a calculation. This allows the calculation of power distributions and flux distributions in a 3D Monte Carlo calculation. Thus, the insight in the neutronics behaviour of an ADS may be substantially increased. Both the criticality analyses and the radiation shielding analyses of an ADS may be performed with MCNP. Finally, MCNP is used for the calculation of various nuclear data sensitivity coefficients. This is an important feature in the ADS design phase, as it allows parametric studies with a 3D Monte Carlo code. The results of sensitivity analyses may be combined with covariance data in order to calculate the uncertainty in calculated responses [21].

The Binary Cascade (BIC) is a new approach to cascade calculations, based on a detailed 3D model of the nucleus, and exclusively based on binary scattering between reaction participants and nucleons within this nuclear model. This feature makes it a hybrid between a classical cascade code, and a quantum molecular dynamics (QMD) model. In particular, BIC agrees better with the data for neutron production, low and medium energy protons, light ion production and isotropic distribution. A very good prediction by BIC is seen for the description of the forward peak spectra in several materials. Tritium production is also well predicted by the model [13]. 
The MCNPX code is a coupling of two previous calculations codes: LAHET and MCNP. MCNPX only needs one input file for both codes and avoids the transfer of large data files. It allows the treatment of transport problems in a large range of energies, from thermal energy, $25 \mathrm{meV}$ to a few $\mathrm{GeV}$. To treat the transport, MCNP uses data deduced of International cross-sections libraries such as ENDF, JEFF, JENDL, etc, after processing them with NJOY or ACER. After running an MCNPX-job, several evaluations can be performed with an auxiliary code, HTAPE3X, to obtain specific information e.g., neutron spectrum, energy deposition, residual nuclei, etc. Most options from older LAHET are used in MCNPX. They include the following models:

- the BERTINI and the ISABEL INC models as in LAHET, and a third INC model: the CEM model;

- the INCL4 model based on Cugnon INC approach;

- a multistage pre-equilibrium model;

- DRESNER and ABLA evaporation model;

- RAL and ORNL fission models;

- a nucleon elastic scattering model;

- a gamma production model [10].

The code SONET being developed for many years in NAS Belarus is dedicated for Monte Carlo simulation of intra- and inter-nuclear cascades in thick targets of arbitrary geometric configuration and material composition. One can calculate transport of nuclei $(\mathrm{A}<16)$, nucleons and pions in the energy region up to $10 \mathrm{GeV}$. Electromagnetic showers are simulated by means of the well-known EGS4 computer code, which is included in the package also. Ionization energy losses, range straggling, pion decay, multiple Coulomb scattering, inelastic interaction of particles (n, p, $\pi^{ \pm}, \gamma$ ) and nuclei with nuclei as well as high energy fission have been taken into account. The code SONET describes nuclear collision products by means of a cascade-exciton model. The model treats a nuclear reaction as a three-stage process i.e., cascade, pre-equilibrium and equilibrium stages unlike well-known two-stage model. The code SONET provides complete description in energy as well as angular and spatial coordinates of the low energy neutrons produced in spallation reactions. Further, low energy neutron transport is described by means of methods developed for radiation shielding and nuclear reactor calculations. The first alternative is represented by the code SYNTES-Q. It allows to calculate neutron transport for subcritical systems in multi-group diffusional approximation in 2D geometry and is based on iterative synthesis method for solving the neutron transport equation with an external source. The second alternative is represented by the well-known code MCNP 4A. One of the versions of the code SONET allows to calculate the evolution of a nucleon-meson cascade in time, which is of interest when considering external pulsed sources. More generally, the code SONET allows calculating the following quantities:

- neutron energy-angle leakage spectra as well as neutron and nuclide yield;

- neutron fluence as well as specified reaction rates for specified geometric cells;

- time evolution of fuel nuclide composition;

- thermal power due to (n, f) and (n, $\gamma$ ) reactions;

- energy production in different geometric cells;

- distribution of fission and capture rates for different nuclides [23].

A Brazilian research group (IFUSP and CBPF) developed the MCMC/MCEF (Multi Collisional Monte Carlo plus Monte Carlo for Evaporation-Fission calculation) model to study nuclear reaction such as spallation. The MCMC and the MCEF model utilize the Monte Carlo approach to describe the intranuclear cascade and the evaporation/fission processes respectively. Their coupling originates the CRISP (Colabora ÇãoRIo-São Paulo) package. The code takes into account the possibility of neutron, proton and alpha particle evaporation and gives information about neutron and proton multiplicity, angular distribution and energy spectra [17].

The NRG Petten and CEA Bruyères-le-Châtel created a computer code system for the simulation and analysis of nuclear reactions named TALYS. The basic objective behind the construction of TALYS is the ability to give a complete description of nuclear reactions that involve neutrons, photons, protons, deuterons, tritons, ${ }^{3} \mathrm{He}-$ and alpha-particles, for target nuclides of mass 12 and heavier [24]. TALYS is a deterministic code which implements various physical models and can quite reliably reproduce various cross-sections in the energy range 1 $\mathrm{keV}-250 \mathrm{MeV}[10]$.

The radiotoxicity of the spallation residue must be accurately known for safety reasons. The concentration limit of the radioactivity can be found based on the ORIGEN2 code. It is found that for the production of the radiotoxic isotopes, the CEM2K and the INCL4K models give the best results with a slight preference for the INCL4K model. Concerning the multiplicity (differential) of neutrons, the Bertini-preequilibrium-dresner model, 
the Isabel-preequilibrium-dresner model, the CEM2K model, and the INCL4-KHS model all reproduce rather well the experimental measurements. With these models, most of the ratios (theory to experiment) stay within $25 \%$ of the discrepancy [25].

Most shielding designs for high-energy accelerators have been performed by using a point kernel method, Moyer model. Moyer model is based on exponential attenuation of neutron dose equivalent for neutrons reaching the equilibrium state after thick shield. This method uses a single built-up factor and an attenuation length. However, for lower energies, the attenuation length depends on the neutron energy and the simple Moyer model is no longer applicable. In the practical neutron shield designs of spallation neutron sources there is a need for quick calculation method such as point kernel code. Neutron attenuation calculations are sometimes made by Monte Carlo method based on intra-nuclear cascade model. However this method is very time consuming and complicated [26].

Besides above there are some existing codes and models also. These are: HERMES [23], HETC (High Energy Transport Code.), NMTC (Nucleon Meson Transport Code), NUCLEUS, SHIELD, GEANT4 (Geometry and tracking), GEM (Generalized Evaporation Model), JAM (Jet AA Microscopic Transport Model), LAQGSM (Los Alamos Quark-Gluon String Model), MARS, TIERCE, BRIEFF [12], TUL (Tamura-Udagawa-Lenske model) [24], Exciton pre-equilibrium decay model and Fermi break-up model [23], KENO-Va (multi-group Monte Carlo code) [27], ALICE, LANCELOT, LILITA, PACE [28] and all of these are used in ADS arrangement for various purposes.

\section{Spallation Wastes and Its Transmutation in ADS}

\subsection{Spallation Wastes}

Continued development of nuclear fission as a source of energy is blocked by three main fears: potential reactor meltdown, proliferation of weaponizable fuel, and unsolved buildup of nuclear waste. As it turns out, the least progress has been made on addressing the waste issue. The concern with nuclear waste is its radioactivityradioactive elements are unstable and can decay into other elements, releasing energy in the process. In some cases this energy can be threatening to humans or other life close enough to be exposed to the radiation. Waste is categorized according to two metrics - the danger it poses, and the lifetime over which it remains dangerous. Some radioactive waste is a byproduct of medical and research efforts, but the majority is produced through nuclear fission in reactors [29].

During the spallation process not only neutrons but also protons and other light nuclei $\left({ }^{2} \mathrm{H},{ }^{3} \mathrm{H}, \mathrm{He}\right)$ are emitted from the excited nuclei. As a consequence, the residual nuclei are not only neutron-poor isotopes of the parent nucleus that decay (mainly by internal $\mathrm{p} \rightarrow \mathrm{n}$ conversion and $\beta^{+}$emission) into lower $\mathrm{Z}$ elements, but these lighter elements are also created directly in the spallation process. Furthermore, there is always a certain probability for a highly excited nucleus to fission and therefore it becomes usual to find medium-heavy and light nuclei in the spectrum of spallation products even for classically "non-fissionable" materials. As well as fission products, the process generates spallation products from the target material, in direct proportion to the energy of the proton beam. Some of these are volatile and will find their way into the cover gas system above the coolant, posing a major maintenance challenge. Their radiotoxicity is likely to exceed that of the fission products in the short term, which is relevant to operation and storage rather than final disposal. Finally, capture reactions can even lead to heavier nuclei than the parent nucleus. It can be seen that, although in small quantities, most elements of the periodic table are generated in a heavy spallation target. The consequences this has on materials properties and possible reactions are not yet well explored [11].

Generally, the Spent Nuclear Fuel (SNF) is the source of nuclear waste in the ADS arrangement (Figure 4). Hazard from the SNF is mainly due to a few chemical elements-plutonium, neptunium, americium, curium; out of which the latter three are classified as minor actinides-MAs and some long-lived fission products such as iodine and technetium at concentration levels of grams per ton and extended burn-ups or use of mixed oxides (MOX) fuel increases the amount of MAs produced [30].

Though the concentration of these nuclides are very minute in comparison to the target material, the hazardous nature stems from the fact that these nuclides have very long half-life and relatively higher radiotoxicity measured in Sv/Bq [13]. In addition, the residual nuclei will contribute to the corrosion and to the radiation damages in the target, accelerator window and structural materials. 


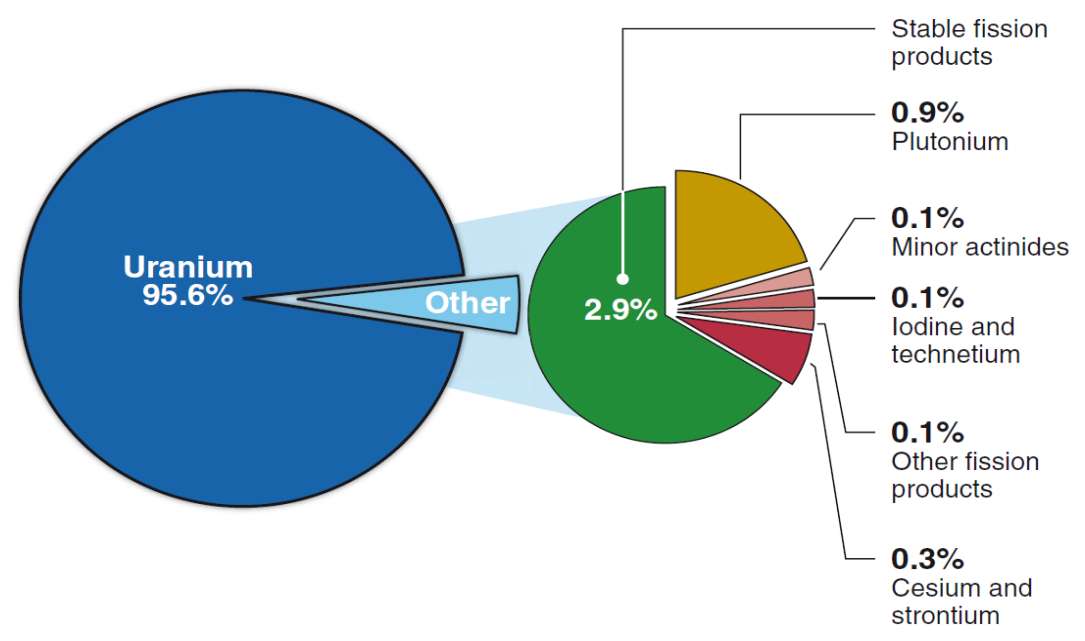

Figure 4. Nuclide distribution of spent nuclear fuel [31].

\subsection{Transmutation in ADS}

The radioactive waste is generated at different stages of the fuel cycle, and can arise in the form of radioactive liquids, gases or solids and with a large spectrum of activity levels. Depending on its activity level and its future management and disposal, it is classified as low, intermediate or high level waste [5]. Management of radioactive waste in an environmentally safe manner is an important issue being addressed by all countries developing a nuclear industry. In many countries it has become a serious political issue attracting intense critical attention of the general public [32].

Treatment, conditioning and long term storage of all kinds of waste are mature technologies and are normally performed at the nuclear facilities where the waste is generated. Storage periods of 50 years or more are not unusual. This allows decay of most radioactive nuclides and flexibility for decisions on disposal. It is the widely held view of technical experts that the method of final disposal for high level wastes (HLW) and spent nuclear fuel (SNF) is likely to be in deep geological repositories. Experience indicates that the time needed to site and develop geological repositories is several decades. Most of the world's spent fuel continues to be stored in reactor pools or dry storage. However, storage represents an interim stage in all spent fuel management strategies, and the final disposal of spent fuel or HLW from spent fuel reprocessing can take decades. Spent fuel continues to accumulate in larger quantities and needs to be stored for longer time periods than initially envisaged (over 100 years). Furthermore, fuel designs are developing to allow much higher burn ups than initially considered in the design basis of many types of storage. Therefore, many different physical, chemical and thermal processes, for example, need to be researched and tested for continued operability, reliability, safety and security of the storage and the spent fuel, and to ensure that the spent fuel can ultimately be safely and securely transported from storage to reprocessing or disposal [5].

The disposal of the wastes arising from nuclear power production is a talkative issue in today's energy world which also discourages the further growth of nuclear power. Research and development (R\&D) work has been going on for a long period of time to find out an efficient and a reliable solution to this problem. As a result of this, today, a technology is working namely ADS which stands for Accelerator Driven System. The concept and design of an accelerator driven system (ADS) is based on the burning up of the wastes produces at the time of power production. The capability of the ADS concept can then be applied to simultaneous burning of the long-lived fission products and actinides in the waste from nuclear power reactors. The goal of burning these fission products is to destroy those long-lived isotopes that, because of their mobility, would be the greatest contributor to radioactive doses from a repository, namely ${ }^{99} \mathrm{Tc},{ }^{129} \mathrm{I}$, and ${ }^{135} \mathrm{Cs}$. These three isotopes constitute about $5 \%$ by weight of the total fission products generated during power production and are the principal contributors to possible long-term risks associated with geologic disposition. If these three isotopes are destroyed internally, the remaining short-lived and stable fission product waste can be confined in engineered storage, perhaps even at the power production site. Maximum burn-up of the fission products requires that they be processed on site. By optimizing the accelerator-driven target or blanket assembly for this burning, the volume and half-life of the 
waste stream can be reduced considerably, thus simplifying long-term storage and secure disposal requirements. Even in this system, where the primary function is to burn radioactive wastes, it is expected that sufficient electrical energy can be produced to pay for the capital and operational costs of the facility [32].

An ADS destroys heavy isotopes contained in the spent fuel from a conventional nuclear reactor-particularly actinides. Here the blanket assembly is actinide fuel and/or spent nuclear fuel. One approach is to start with fresh spent fuel from conventional reactors in the outer blanket region and progressively move it inwards. It is then removed and reprocessed, with the uranium recycled and most fission products separated as waste. The actinides are then placed back in the system for further incineration. The whole process is done following a flow chart given in Figure 5.

Commercial application of partitioning and transmutation (P\&T), which is attractive particularly for actinides, is still a long way off, since reliable separation is needed to ensure that stable isotopes are not transmuted into radioactive ones. New reprocessing methods would be required, including electrometallurgical ones e.g., pyroprocessing. The cost and technology of the partitioning together with the need to develop the necessary highintensity accelerators seems to rule out early use. An NEA study showed that multiple recycling of the fuel would be necessary to achieve major (e.g. 100-fold) reductions in radiotoxicity, and also that the full potential of a transmutation system can be exploited only with commitment to it for 100 years or more.

Another area of current interest in the use of ADSs is in their potential to dispose of weapons-grade plutonium, as an alternative to burning it as mixed oxide fuel (MOX) in conventional reactors. Two alternative strategies are envisaged: the plutonium and minor actinides being managed separately, with the latter burned in ADSs while plutonium is burned in fast reactors; and the plutonium and minor actinides being burned together in ADSs, providing better proliferation resistance but posing some technical challenges. Both can achieve major reduction in waste radiotoxicity, and the first would add only 10\% - 20\% to electricity costs (compared with the once-through fuel cycle) [8].

Figure 6 illustrates the effectiveness of transmutation in reducing the overall radiotoxicity of the system. For example, ${ }^{245} \mathrm{Cm}$ with half-life of 8500 years is fissioned using a neutron. The fission products ${ }^{110} \mathrm{Pd}$ is stable and ${ }^{134} \mathrm{Cs}$ has a comparatively shorter half-life of two years.

Besides the above, another problem is the reactor itself. As power reactors reach the end of their life cycles they need to be decommissioned. As some parts of the reactors are radioactively contaminated, they will need to be dismantled in a controlled way and the radioactive waste taken care of. The timing of the dismantling is dependent on several factors, for example, radiation protection considerations, availability of funding and availability of disposal facilities. The radioactive waste from decommissioning is mostly low and intermediate level and can be handled and disposed of accordingly. For some of the components that are very large, special approaches, such as intact disposal, have been successfully used [5].

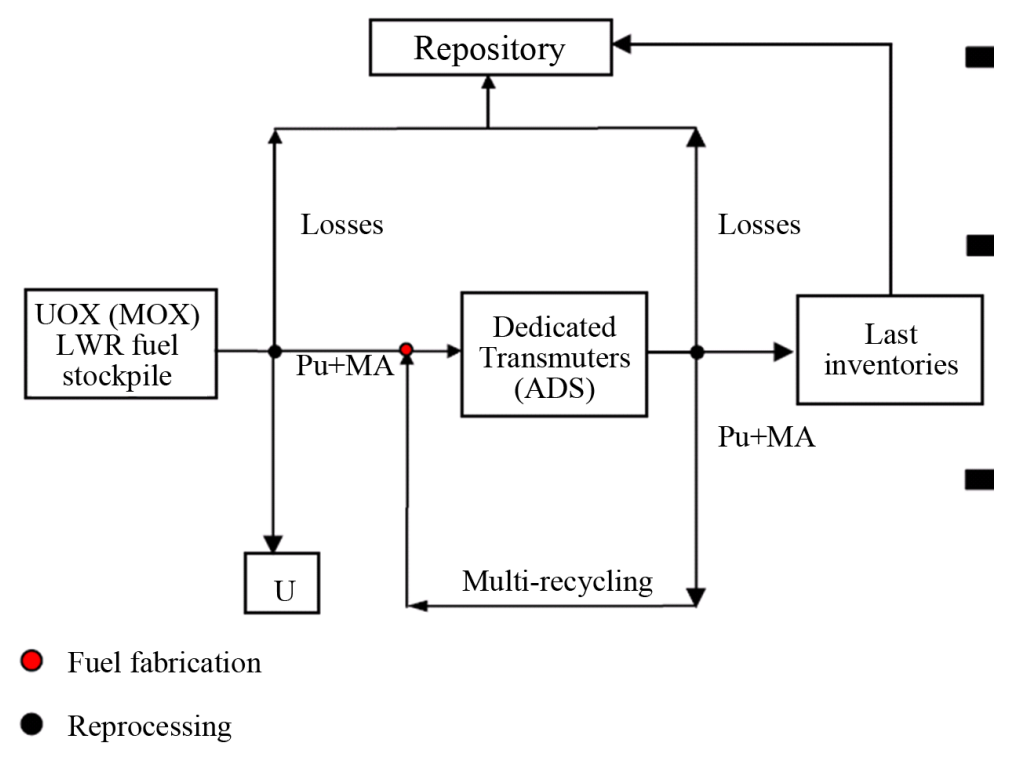

Figure 5. Partitioning and transmutation of nuclear wastes in ADS [30]. 


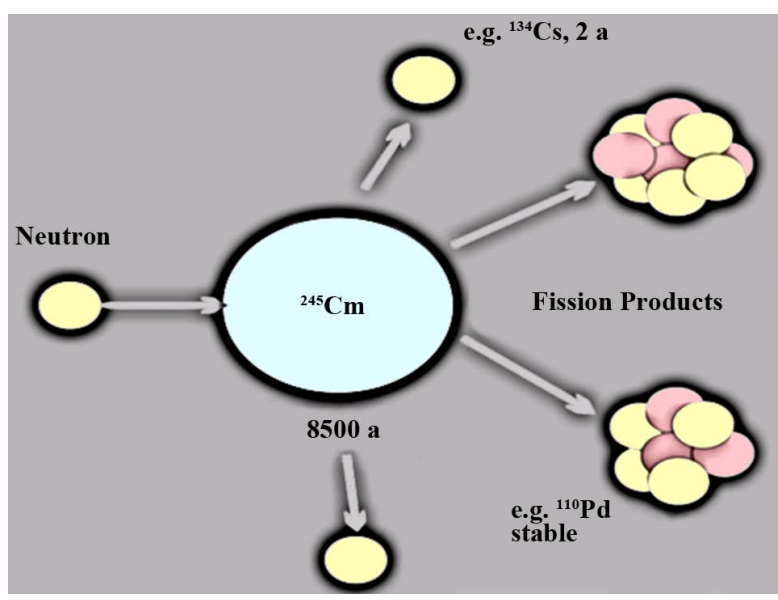

Figure 6. Transmutation of ${ }^{245} \mathrm{Cm}$ isotope using neutron in ADS [13].

\section{Conclusions}

Energy is vital to human civilization and underpins national security, economic prosperity, and global stability. The first line is more than appropriate if it is of green in nature. Therefore, standing on different limitations of the existing non-renewable and renewable sources of electric energy generation as the basis, this paper includes the scopes of nuclear source as a green, efficient and reliable one.

Although nuclear power already has inherently low GHG emissions, future emissions would be even lower due to more energy efficient production of enriched uranium, improved nuclear fuels and reactors that allow greater utilization, and extended life times for NPPs that reduce the need to build new facilities. Moreover, it offers unique benefits as it is the only existing technology with capability for major expansion that can simultaneously provide stability for base-load electricity, security through reliable fuel supply, and environmental stewardship by avoiding emissions of greenhouse gases and other pollutants. Furthermore, it has proven reliability (greater than 90 percent capacity factor), exemplary safety, and operational economy through improved performance [2]. Despite all of the above, the public acceptability of nuclear power is adversely affected by negative perceptions concerning its safety, links with proliferation, and the radiotoxicity of its waste. Therefore for future efficient growth of nuclear power, it will be necessary to satisfactorily address the troublesome issue of disposal of the nuclear waste. Therefore, nuclear waste disposal is certainly an urgent and important issue to be tackled to ensure that future growth of this sector is an important finding of this study and an ADS is, therefore, quite relevant for further growth of nuclear power. In many respects, accelerator-driven systems (ADS) are worth pursuing. By producing electricity, they can contribute to the world's growing energy needs, and by incinerating plutonium and highly radioactive wastes, they can contribute to the goals of environmental protection and safe waste management. Beyond this, there is the promise of systems with the goal to burn weapons plutonium and to incinerate spent nuclear fuel, including its major fission products, from commercial nuclear power plants [32].

But from this study it is found that all of the above can be achieved only if it is possible to ensure a reliable and successful ADS design. However a successful ADS design requires an efficient spallation technique with all its peripherals. Moreover, the efficiency of spallation reactions basically depends on the beam energy and on the target characteristics. Available information regarding the beam energy is more or less sufficient to carry out spallation reactions effectively but most of the problems lie in the target module. The outcome of the spallation reactions sharply varies with different parameters, for example, target material and its composition, atomic mass, size, thickness, etc., involved in the target. Also, appropriate coding systems play a vital role in theoretical analysis. It is found that most of the existing codes used for analyzing data show a discrepancy of about $20 \%-25 \%$ with the experimental one. Also, the codes take their inputs from data libraries whereas the existing high energy data are not sufficiently available for ADS applications. Hence this study says that there is a strong need of further and specific research in designing the target characteristics, improving the coding systems (highly capable of minimizing the discrepancy with experimental results), and high energy reactions (to enrich nuclear data library) so that the ADS would be able to show its potential, in a greater domain, to provide a more sustainable, cost-effective and safer form of nuclear energy in the future. 


\section{References}

[1] Lackner, K.S. (2010) Comparative Impacts of Fossil Fuels and Alternative Energy Sources, Issues in Environmental Science and Technology 29, Carbon Capture: Sequestration and Storage, Royal Society of Chemistry. www.rsc.org

[2] (2008) A Sustainable Energy Future: The Essential Role of Nuclear Energy. https://inlportal.inl.gov/.../NLDC_ltr_Future_of_Nuclear-Final.pdf

[3] (2010) Meeting the Energy Challenges of the Future-A Guide for Policymakers. National Conference of State Legislatures, a Forum for America's Ideas. www.ncsl.org

[4] http://www.renewable energysources.com/

[5] (2011) International Status and Prospects of Nuclear Power, 2010 Edition, International Atomic Energy Agency Vienna. www.iaea.org/Publications/Booklets/NuclearPower/np10.pdf

[6] MIT Study on the Future of Nuclear Power, the Future of Nuclear Power-Over View and Conclusions. Chapter \#1. http://web.mit.edu/nuclearpower/pdf/nuclearpower-ch1-3.pdf

[7] Kapoor, S.S. (2002) Accelerator-Driven Sub-Critical Reactor System (ADS) for Nuclear Energy Generation. PramanaJournal of Physics, 59, 941-950. http://dx.doi.org/10.1007/s12043-002-0143-z

[8] http://www.world-nuclear.org/info/Current-and-Future-Generation/Accelerator-driven-Nuclear-energy/

[9] Kowalczyk, A. (2007) Proton Induced Spallation Reactions in the Energy Range 0.1 - 10 GeV. A Doctoral Dissertation Prepared at the Institute of Nuclear Physics of the Jagiellonian University, Submitted to the Faculty of Physics, Astronomy and Applied Computer Science at the Jagiellonian University, Cracow, 1-162.

[10] Majerle, M. (2008) Monte Carlo Methods in Spallation Experiments. Report for the PhD State Exam, Czech Technical University in Prague, 1-55.

[11] Bauer, G.S. (1998) Physics and Technology of Spallation Neutron Sources. Lecture Notes of a Course Given at the Frederic Jolliot Summer School in Cadarache, France; Intended for Publication, in Revised Form, in a Special Issue of Nuclear Instruments and Methods A, 1-44.

[12] Krása, A. (2010) Spallation Reaction Physics. Manuscript for the Lecture "Neutron Sources for ADS” for Students of the Faculty of Nuclear Sciences and Physical Engineering at Czech Technical University, Prague, 1-61.

[13] Shetty, N.V. (2013) Study of Particle Transport in a High Power Spallation Target for an Accelerator-Driven Transmutation System. Master’s Thesis, RWTH Aachen University, Aachen, 1-138.

[14] Kadi, Y. and Revol, J.P. (2005) IAEA/ICTP Workshop on Technology and Applications of Accelerator Driven Systems (ADS). ICTP, Trieste.

[15] Armbruster, P. and Benlliure, J. (2001) Basic Nuclear Data at High and Intermediate Energy for Accelerator-Driven Systems. http://www.nupecc.org/iai2001/report/A6.pdf

[16] Wade, D.C. (2001) Safety Considerations in Design of Fast Spectrum ADS For Transuranic or Minor Actinide Burning: A Status Report on Activities of the OECD/NEA Expert Group. Overview Paper, 95-118. www.researchgate.net/.../237451934

[17] Mongelli, S.T., Maiorino, J.R., Anéfalos, S., Deppman, A. and Carluccio, T. (2005) Spallation Physics and the ADS Target Design. Brazilian Journal of Physics, 35, 894-897. http://dx.doi.org/10.1590/S0103-97332005000500048

[18] Zhang, Y.L., Zhang, X.C., Qi, J., Wu, Z. and Yang, L. (2013) Study on the Parameters of the ADS Spallation Target. Journal of Physics: Conference Series, 420, Article ID: 012064. http://dx.doi.org/10.1088/1742-6596/420/1/012064

[19] OECD (2005) Accelerator and Spallation Target Technologies for ADS Applications, Nuclear Energy Agency, Organization for Economic Co-Operation and Development. A Status Report, NEA No. 5421.

[20] Leray, S., Boudard, A., David, J.C., Donadille, L., Villagrasa, C. and Volant, C. (2005) Impact of High Energy Nuclear Data on the Radio-Protection in Spallation Sources. Radiation Protection Dosimetry, 115, 242-246. http://dx.doi.org/10.1093/rpd/nci086

[21] Klippel, H.T. (2003) ADS Activities in the Netherlands. IAEA-TECDOC-1365, 166-175.

[22] Khandaker, M., Mank, G., Mengoni, A., Otuka, N., David, J.-C., Leray, S., et al. (2010) Codes and Data for Spallation Sources, Benchmark of Nuclear Spallation Models. http://scholar.google.com

[23] Chigrinov, S.E., Kievitskaia, A.I., Rakhno, I.L., Serafimovich, I.G., Rutkovskaia, C.K., et al. (2003) Research on Accelerator Driven Systems in the National Academy of Sciences of Belarus. IAEA-Tecdoc-1365, 78-91.

[24] Koning, A., Beijers, H., Benlliure, J., Bersillon, O., et al. (2002) A European Nuclear Data Programme for AcceleratorDriven Systems. Seventh Information Exchange Meeting on Actinide and Fission Product Partitioning and Transmutation, 723-734. https://www.oecd-nea.org/pt/docs/iem/jeju02/session5/SessionV-05.pdf

[25] Aoust, T., Malambu, E.M. and AïtAbderrahim, H. (2005) Importance of Nuclear Data for Windowless Spallation Tar- 
get Design. In: Proceedings of the International Conference on Nuclear Data for Science and Technology, American Institute of Physics, College Park, 1572-1575.

[26] Fragopoulou, M. (2006) Shielding around Spallation Neutron Sources. Journal of Physics: Conference Series, 41, 514518. http://dx.doi.org/10.1088/1742-6596/41/1/058

[27] Magill, J. and Peerani, P. (1999) (Non-) Proliferation Aspects of Accelerator Driven Systems. Journal de Physique IV, 9, Pr7-167.

[28] Benlliure, J. (2006) Spallation Reactions in Applied and Fundamental Research. Lecture Notes in Physics, 700, 191238. http://dx.doi.org/10.1007/3-540-33787-3_5

[29] Ray, J. (2011) Accelerator Transmutation of Waste. Submitted as Coursework for Physics 241, Stanford University, Stanford.

[30] OECD (2006) Physics and Safety of Transmutation Systems. Technical Report, NEA No. 6090.

[31] GAO (2011) Nuclear Fuel Cycle Options. Technical Report GAO-12-70, United States Government Accountability Office, Washington DC.

[32] Arkhipov, V. (1997) Future Nuclear Energy Systems: Generating Electricity, Burning Wastes. IAEA Bulletin, 39, 30-33. 